\title{
Locally Advanced Breast Cancer-A Narrative Review of Management in the Indian Scenario
}

\author{
Manjula Rao ${ }^{1}$ Manikandan Dhanushkodi² \\ ${ }^{1}$ Consultant Breast Oncosurgeon, Breast CMT, Apollo Proton Cancer \\ Centre, Tharamani, Chennai, Tamil Nadu, India \\ ${ }^{2}$ Department of Medical Oncology, Cancer Institute (WIA), Adyar, \\ Chennai, Tamil Nadu, India
}

\author{
Address for correspondence Manjula Rao, MBBS, MS, Apollo Proton \\ Cancer Centre, Tharamani, Chennai, Tamil Nadu, 600096, India \\ (e-mail: docmanjrao@gmail.com).
}

\begin{abstract}
Keywords

- locally advanced breast cancer

- neoadjuvant systemic therapy

- breast conservation in $L A B C$

- cost effectiveness

- multimodal management

Locally advanced breast cancer ( $L A B C$ ) forms a significant proportion of the total breast cancer (BC) burden in the country. It is a heterogeneous entity, encompassing a variety of presentations indicative of advanced disease. The standard of care for the treatment of $L A B C$ is multimodal, involving chemotherapy, surgery, radiation, and/or hormonal therapy. With improved understanding of the biology of BC, addition of newer agents of systemic therapy to our armamentarium, advances in surgical techniques and radiation delivery, clinicians are now able to tailor treatment according to the individual and consider a more conservative approach to management (in contrast to a mandatory radical mastectomy, decades ago), disease biology, and stage permitting with better survival outcomes. Here, we review all the treatment modalities and options available to aid a clinician in formulating an optimal treatment plan for a patient presenting with $L A B C$ and also how best to tailor the treatment to enable cost effectiveness in the Indian setting.
\end{abstract}

\section{Introduction}

Breast cancer (BC) has emerged as the most frequently occurring cancer in women in India and worldwide, affecting 2.1 million women each year. ${ }^{1}$ India alone is accounted for $3,162,331$ cases (23.8\%) of the total prevalence

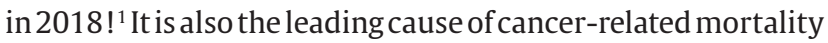
in women (626,679 women, accounting for $15 \%$ mortality). ${ }^{1}$ The urban $\mathrm{BC}$ registries in India show an increasing trend in the incidence of the disease, owing to the adoption of a more westernized lifestyle and food habits, by the inhabitants. ${ }^{2}$

Early detection plays a very important role in improving the outcomes and survival of BC. A total of $45.7 \%$ of patients in India present to clinicians at advanced stages of the disease, at first visit. ${ }^{2}$ General lack of awareness among the population regarding cancer, self-breast examination (SBE), dearth of screening programs, personal and social factors such as stigma and taboos among society concerning cancer

DOI https://doi.org/ 10.1055/s-0041-1732785 ISSN 0971-5851 as a disease and the breast as an organ, the general disregard toward woman's health in rural or traditional patriarchal societies, and financial constraints among others, are all reasons that contribute to the patient desisting from a consultation at early stages. ${ }^{2,3}$ The Indian population is known to present with breast cancer at least a decade younger, in comparison to women in the West, and is known to be more aggressive. ${ }^{2}$

\section{Definition of Locally Advanced Breast Cancer}

The definition of locally advanced BC (LABC) has seen continuous modifications over the last four to five decades. It includes a heterogeneous group of breast tumors with extensive locoregional spread that may be operable or inoperable without any clinicoradiological evidence of metastasis. The eighth edition of the American Joint Committee on Cancer (AJCC) cancer staging manual

(C) 2021. Indian Society of Medical and Paediatric Oncology. This is an open access article published by Thieme under the terms of the Creative Commons Attribution-NonDerivative-NonCommercial-License, permitting copying and reproduction so long as the original work is given appropriate credit. Contents may not be used for commercial purposes, or adapted, remixed, transformed or built upon. (https://creativecommons.org/licenses/by-nc-nd/4.0/). Thieme Medical and Scientific Publishers Private Ltd. A-12, Second Floor, Sector -2, NOIDA -201301, India 
groups under LABC, T3 tumors ( $>5 \mathrm{~cm}$ ), with or without skin or chest wall involvement, alone or together, inflammatory BCs (IBC), matted ipsilateral and/or fixed axillary lymph nodes (AxLNs), ipsilateral supraclavicular lymph nodes (SCLNs), and/or internal mammary lymph nodes (IMLNs) without distant metastases. ${ }^{4}$ IBC, classified as T4d, in the AJCC staging system, is a distinct clinicopathological entity, characterized by diffuse, brawny induration of the skin of the breast with an erysipeloid edge, usually without an underlying detectable mass. They are the most aggressive forms of LABC that are distinct from the other T4 lesions, hallmarked by rapid progression and worse prognosis, with a 5-year survival of less than $45 \%{ }^{5,6}$ This clinical presentation of IBC occurs due to involvement of the dermal lymphatics by the cancer cells; and though the demonstration of tumor cells by a biopsy in the dermis of the breast is considered confirmatory, it is not compulsory for diagnosis of IBC. ${ }^{6}$

\section{Investigating a Case of Locally Advanced Breast Cancer}

The workup for LABC begins with a triple assessment, consisting of history and physical examination, followed by appropriate imaging and a core biopsy for confirmation (-Fig. 1). The clinical findings must be documented in detail to enable accurate response assessment. Many clinicians prefer to do the same with a diagrammatic representation to avoid any confusion in deciphering the documented details when multiple clinicians are involved ( - Fig. 2 ).

Bilateral mammography (MMG) is the preferred imaging modality with ultrasonography (USG) used as an adjunct for better characterization of the lesion and assessment of the AxLN. In certain patients with very dense breasts, mammographically occult tumors or those who are desirous of breast conservation, magnetic resonance imaging (MRI) of

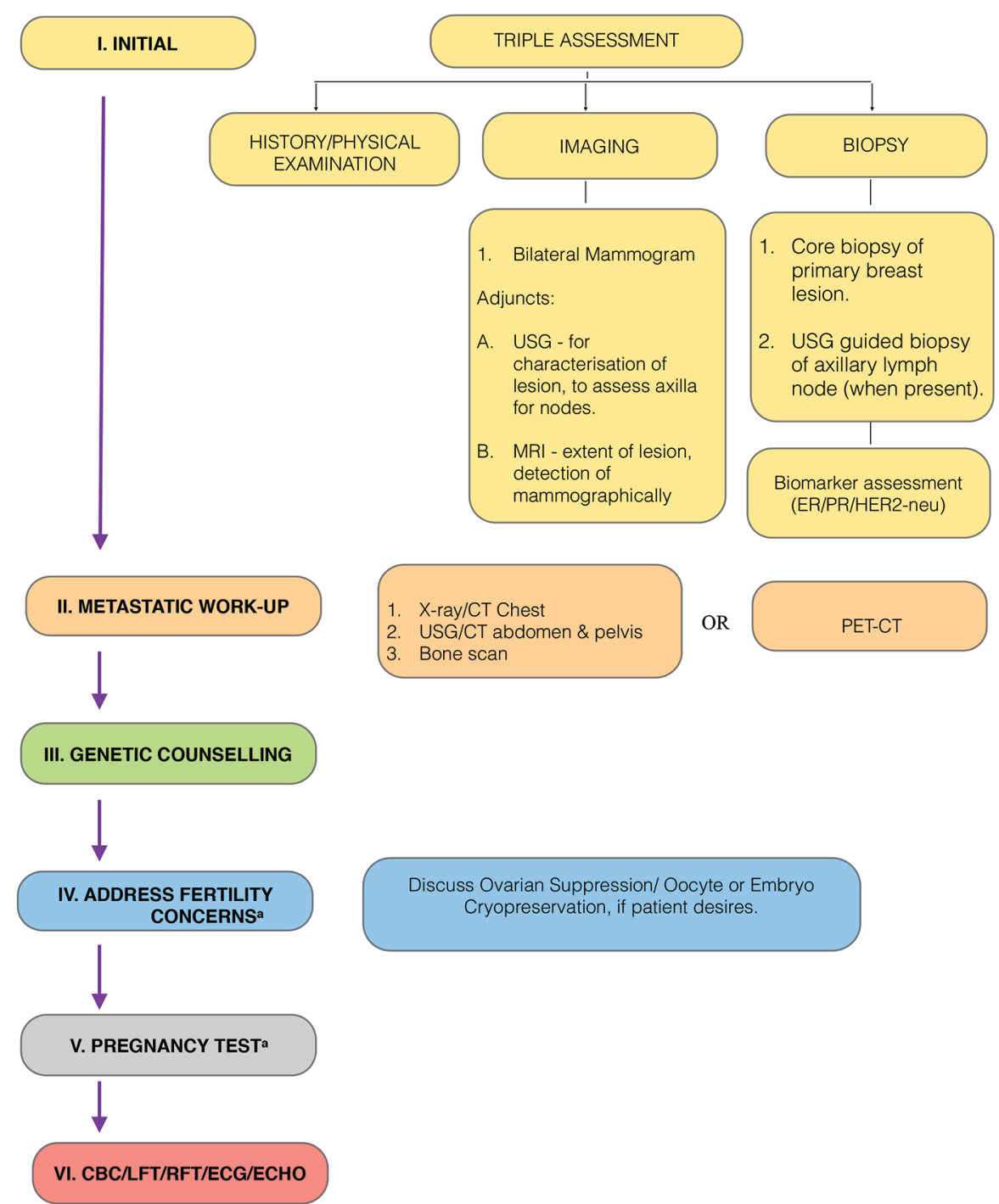

Fig. 1 Investigations to diagnose and work-up a patient with locally advanced breast cancer. aln premenopausal women. CBC, complete blood count; CT, computerized tomography; ECG, electrocardiogram; ECHO, echocardiogram; ER, estrogen receptor; HER2-neu, human epidermal growth factor receptor 2; LFT, liver function tests; MRI, magnetic resonance imaging; PET, positron emission tomography; PR, progesterone receptor; RFT, renal function tests; USG, ultrasonogram. 


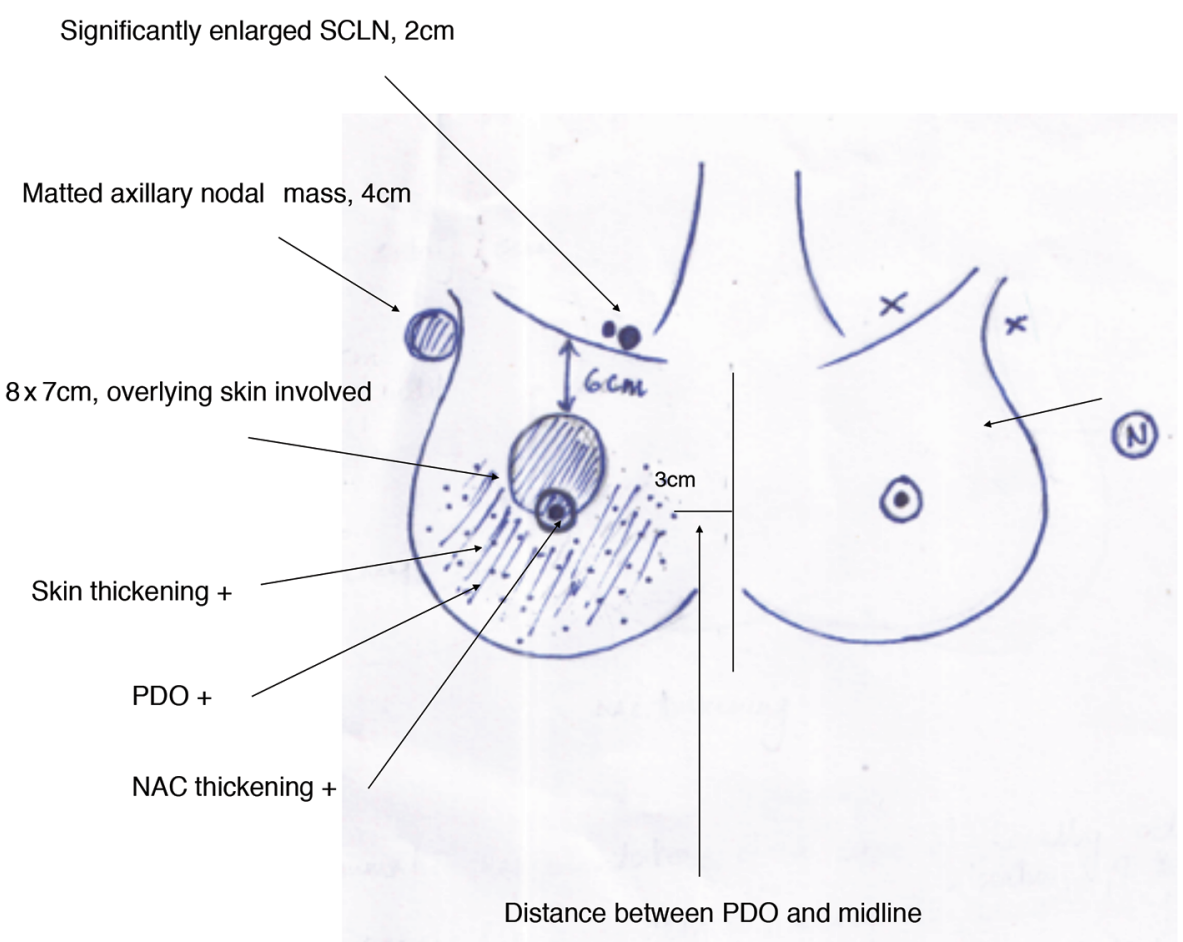

Fig. 2 An example of a diagrammatic representation of a case of locally advanced breast cancer which helps clinicians assess the response of the disease to systemic therapy or detect progression if any. The above figure demonstrates a breast lump with involvement of overlying skin, nipple areola complex (NAC) thickening, peau d'orange (PDO), skin thickening over the surrounding breast skin, and description of the regional lymphadenopathy as well. It also marks the distance between the lump and the PDO with surrounding recognizable bony landmarks, so as to aid the surgeon in assessing response and to plan the volume of excision as well. SCLN, supraclavicular lymph node.

the breasts may be used as an adjunct. Confirmation of the diagnosis by a core biopsy is preferred over a fine-needle aspiration cytology (FNAC), since it will yield more material to demonstrate invasion and status of biomarkers, that is, estrogen receptor (ER), progesterone receptor (PR), and human epidermal growth factor receptor 2 (HER2/neu). A guided biopsy or FNAC of any suspicious AxLN may be done to confirm metastatic involvement for staging, with a clip placed in situ in the same sitting to ensure that the positive node has been removed at the time of definitive surgery. If the patient's family history is indicative of a higher risk for hereditary BC (HBC), then genetic counseling is advised. A metastatic workup must be done for the completion of staging, consisting of an assessment of the chest by computerized tomography (CT) or an X-ray, abdominal assessment by CT or USG, and assessment of the bones by a bone scan or fluoride positron emission tomography (PET). Fluorodeoxyglucose (FDG) PET-CT is usually recommended if the standard investigations are equivocal or nonconclusive but suspicious. ${ }^{7}$

\section{Management of Locally Advanced Breast Cancer}

Management of LABC has evolved over the last six to seven decades from a single modality of treatment (radical surgeries or high doses of radiation) to multimodal management by combining chemotherapy, surgery, and radiation which has drastically improved the survival outcomes in these patients.
Neoadjuvant systemic therapy (NST), followed by surgery, adjuvant chemotherapy, locoregional radiotherapy (RT), and/ or hormonal therapy (HT), is now considered the gold standard in LABC treatment ${ }^{8}(-$ Fig. $\mathbf{3})$.

\section{Chemotherapy}

The survival benefits of adjuvant chemotherapy in the treatment of BC have been well documented in early literature. ${ }^{9}$ Use of induction chemotherapy or NST was initiated in the latter half of the 20th century with multiple encouraging reports showing downstaging of the disease, rendering inoperable $\mathrm{LABC}$ resectable and/or downsizing the primary in operable $\mathrm{LABC}$, enabling breast conservation, if feasible, and if the patient so desires. It allows us to study the in vivo response of the tumor to the treating agent, providing us insight into disease biology which would thereby allow us to tailor treatment accordingly. There was no difference in survival outcomes between neoadjuvant and adjuvant chemotherapy..$^{10}$ The achievement of pathological complete response ( $\mathrm{pCR}$ ), which means complete clinical and pathological disappearance of the disease is an indication of favorable biology and outcomes.

Chemotherapy may be administered through a peripherally inserted central catheter (PICC) or a chemo port which is a good alternative to repeated intravenous line insertions and safeguard against the risk of extravasation. Addressing fertility concerns of the patient and a pregnancy test in women of childbearing potential are prerequisites to beginning NST. 


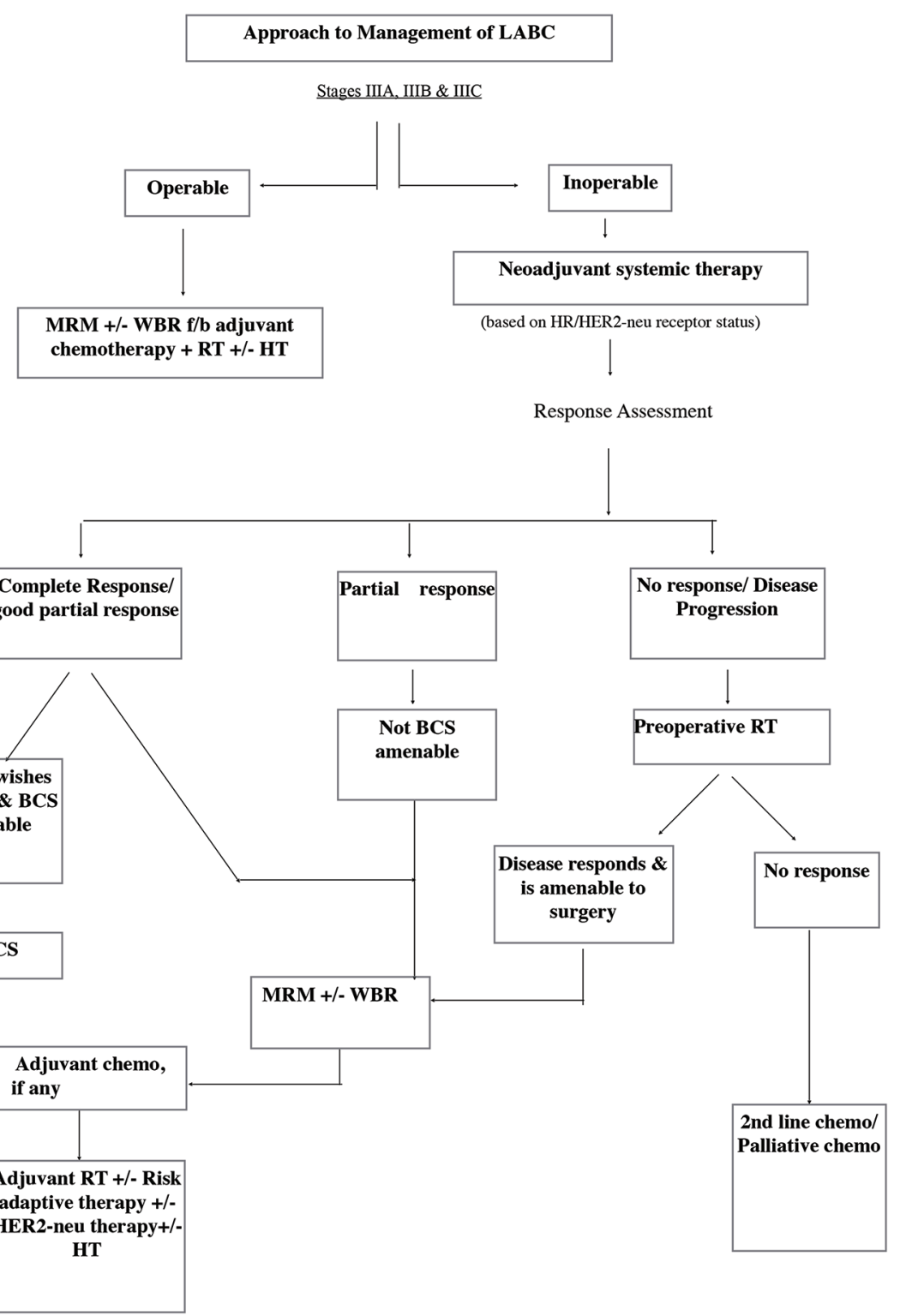

Fig. 3 Algorithm to guide in sequence of treatment in a case of locally advanced breast cancer (LABC). BCS, breast conservation surgery; HER2, human epidermal growth factor receptor 2; HT, hormone therapy; MRM, modified radical mastectomy; RT, radiotherapy; WBR, whole breast irradiation.

Starting a premenopausal patient on gonadotropin-releasing hormone $(\mathrm{GnRH})$ analogs before initiation of chemotherapy and its continuation throughout chemotherapy has shown to prevent ovarian failure, hence avoiding the ill effects of attaining premature menopause and also doubles the chances of a successful pregnancy. ${ }^{11}$ Those desirous of childbearing in the future should be counseled regarding options of embryo or oocyte cryopreservation.

Chemotherapeutic regimens used in the treatment of $\mathrm{BC}$ are tailored as per the biological tumor receptor (ER/PR/HER2) status, based on which tumors may be classified into subgroups such as luminal A (ER/PR positive, HER2 negative, with low-risk pathological features), luminal B (ER/PR positive with high-risk pathological features), HER2 overexpressing tumors (ER/PR negative and HER2-positive), and basal-type (subtype of triple-negative $\mathrm{BCs}[\mathrm{TNBC}]$ where hormone receptors are not expressed). The response to NST and overall outcome is determined by the disease subset. Luminal B, HER2 positive, and TNBC subtypes tend to be more aggressive biologically but are also more responsive to chemotherapy and have a higher chance of attaining PCR.

Anthracycline-based regimens have proven to be superior in the treatment of BC, while the node-positive subset benefitted from the addition of taxanes to their regimens. ${ }^{12}$ There was no difference in outcomes when taxanes were given preoperatively, sequential to anthracyclines, or postoperatively, but completion of chemotherapy preoperatively resulted in significantly higher pCR rates. ${ }^{12}$ In clinical practice typically, four cycles of adriamycin-cyclophosphamide (AC)/epirubicin-cyclophosphamide (EC) are administered, followed by four 3-week cycles of taxanes (paclitaxel/docetaxel). Studies have shown that accelerated or dose-dense chemotherapy, 
wherein the chemotherapy is delivered at 2-week instead of 3-week cycle, has a lower risk of locoregional recurrence (LRR) and better survival. ${ }^{13}$ Patients with HER2-positive disease tend to have improved survival outcomes with the addition of HER2-targeting agents, such as trastuzumab, and the newer monoclonal antibody pertuzumab. These patients may be initiated on treatment, with six cycles of nonanthracycline therapy, TCHP (docetaxel-carboplatin-trastuzumab-pertuzumab), ${ }^{14}$ and continuation of HER2-targeted agents postoperatively, as maintenance therapy for a year. ${ }^{15}$ Pertuzumab may be omitted from the regimen for those patients who cannot afford it. Alternatively, they can receive anthracycline-based chemotherapy with taxanes and trastuzumab administered sequentially or vice versa. The presence of residual disease in the surgical specimen after completion of all chemotherapy allows us the opportunity to administer additional chemotherapy in certain patient subsets which is called risk-adapted therapy. Patients with HER2-positive BC who do not achieve pCR can be treated with adjuvant TDM-1 (ado-trastuzumab emtansine) for 12 cycles, ${ }^{16}$ and those with TNBC who do not achieve pCR can be treated with adjuvant capecitabine for 6 cycles. ${ }^{17}$

Neoadjuvant HT (NAHT) may be considered in patients with HR-positive and HER2-negative BC; it offers a response similar to NST with decreased toxicity. ${ }^{18}$ It is a safer alternative in elderly patients, those with comorbid illnesses, poor performance status, and those unwilling to accept the side effects of chemotherapy; one major drawback being the prolonged duration of NAHT for the desired downsizing of the tumor.

\section{Assessment of Clinical Response to Neoadjuvant Systemic Therapy}

The clinical response must be assessed and recorded before every cycle of chemotherapy by the treating clinicians. Imaging modalities such as MMG/USG or MRI may be used as an adjunct to clinical examination, especially when the patient is desirous of breast conservation, with percutaneous marker placement (titanium clip and etcetera) to guide the surgeons during surgery in case of complete clinical and radiological response of the tumor. A fraction of patients may fail to respond or progress during chemotherapy. In this situation, options include either changing the chemotherapeutic regimen or surgical intervention (so as not to lose the window of opportunity for surgery). In case, the disease remains inoperable, one can change to the second line of chemotherapy. It indicates poor biology, and clinicians may have to abandon hopes of a complete locoregional cure in this scenario. Setups with resource constraints can choose to tattoo the skin over the tumor or take a stitch at the skin over the center of the tumor, so that the healed scar serves as a marker.

\section{Surgical Management}

In the late 19th century, William Halsted proposed his theory of centrifugal spread of $\mathrm{BC}$ with extension to the regional lymphatics, if left untreated, which was embraced by the medical community for nearly a century when radical mastectomy
(RM; en bloc resection of the breast, AxLN, chest-wall musculature, and even IMLN in some cases) was considered the standard of care. ${ }^{19}$ This mindset began shifting when Mc Whirter suggested that one could attain equivalent survival with a conservative surgical approach and the addition of radiation to the treatment protocol. ${ }^{20}$ Fisher's theory suggesting that $\mathrm{BC}$ was a systemic disease in which the malignant cells would have probably metastasized even before the diagnosis, revolutionized BC management. ${ }^{21}$ Evidence supporting conservative surgical techniques that decreased morbidity and had equivalent survival in the era of multimodal management, helped facilitate the switch from RM to modified radical mastectomy (MRM) which involves resection of the involved breast and the ipsilateral AxLN.22 There is ongoing research to identify the patient subset in whom limited axillary dissection may be done instead of the routine, morbid axillary clearance without adversely affecting survival.

\section{Breast Conservation Surgery in Locally Advanced Breast Cancer}

Breast conservation therapy (BCT; breast conservation surgery [BCS] followed by adjuvant RT) has long been established as the standard of care in the treatment of early BC, following the results from numerous prospective randomized controlled trials (RCTs), demonstrating no significant difference in overall survival (OS) between BCT and MRM. ${ }^{23,24}$ With multidisciplinary management coming to the fore and NST allowing downsizing of tumors, various disease management groups attempted to investigate the safety of BCT, in patients with LABCs as well. ${ }^{25}$ BCS following NST, in the early days, showed high rates of ipsilateral breast tumor recurrences (IBTRs) and LRRs and was looked at with caution. Closer observation attributed this to the omission of surgery or adjuvant RT by certain study groups. ${ }^{26,27}$ Multiple studies conducted since then have proven the safety of BCT following NST in more advanced presentations, provided patients have been appropriately selected. ${ }^{28-30}$ Advanced nodal disease at presentation, size of residual disease $>2 \mathrm{~cm}$, multifocality, presence of lymphovascular invasion (LVI) in the final histopathology, and margin positivity following BCS are few factors found to be associated with increased IBTR and LRR. ${ }^{31}$ Parmar et al demonstrated a superior 3-year disease-free survival (DFS) and lower LRR in patients who underwent BCT after NST. They have attributed this to an inherent selection bias, as responders to NST were randomized to the BCT arm, whereas nonresponders underwent MRM. ${ }^{28}$

For BCS post-NST, the residual disease guided by the marker-placed pretreatment, is excised with adequate margins which may be assessed intraoperatively by a frozen section (FS) examination for those with the facility. Those without, must await the postsurgery histopathology. In case of positive or doubtful margins, reexcision of margins or mastectomy may be considered, depending on the pathology, multi-disciplinary team discretion and informed patient decision. No attempt is made to excise the pre-NST volume of tumor. Clips are placed at the cavity base for identification to enable adjuvant radiation boost. The cavity is either closed primarily or by using oncoplastic techniques for improved 
cosmesis. Adequate margin for invasive breast carcinoma has been defined as "no tumor on ink"32; whether this can be extrapolated to BCS in patients with LABC following NST is debatable. Diligence is due in this regard and taking a wider margin may be considered in those patients with LVI, nonuniform tumor shrinkage, etc.

Earlier, BCS was restricted to tumors less than $5 \mathrm{~cm}$. This is no longer an absolute contraindication with the growing popularity and increasing utilization of oncoplastic techniques for favorable cosmetic outcomes. BCT is contraindicated in patients with IBC, despite the complete response of the skin and tumor to NST. Recently, Silverstein et al described "Extreme Oncoplasty," which allows breast conservation in those patients with extensive disease in the breast who would have otherwise been offered mastectomy by most breast specialists. ${ }^{33}$ This is also being explored in Indian centers. ${ }^{34}$

\section{Surgery in the Case of Internal Mammary Lymph Node Involvement}

The IMLN basin is one of the primary lymphatic draining sites in the breast. Enough and more evidences in literature show that BC patients with IMLN involvement have a worse prognosis. ${ }^{35}$ IMLN involvement in BC was extensively studied by Handley and Thackray ${ }^{36}$ in the middle1900s and surgeons actively resected them during RM and called it extended RM. Studies, however, failed to show any survival benefit from such extensive dissections ${ }^{37-40}$ were hence abandoned, and a more conservative surgical approach was adopted.

\section{Adjuvant Radiation Therapy}

From being used solo in the treatment of inoperable $\mathrm{BC}$, to being used in the adjuvant setting, the utility of RT in BC treatment has evolved greatly. Irradiating the chest wall and regional lymph nodes after mastectomy reduce subsequent LRR and death from BC, as evidenced by multiple studies. ${ }^{41-44}$ Earlier, outdated equipment, techniques, and imprecise RT delivery resulted in increased morbidity, hence did not translate into improved survival outcomes. These issues have been resolved with evolving technology and better understanding of the disease.

Postmastectomy RT (PMRT) is now recommended in patients with features of high risk of recurrence, node positivity, tumors more than $5 \mathrm{~cm}$, SCLN involvement, and skin and/or chest wall involvement. ${ }^{45}$ Adjuvant RT is recommended even in those patients who have achieved pCR, since it is advisable to determine the adjuvant treatment based on their stage at presentation. ${ }^{46}$ PMRT includes irradiation of chest wall (including mastectomy flaps, scar, and drain sites); regional lymph nodes, that is, SCLN; portion of the axilla that is not addressed surgically; and IMLN, in a standard dose of 45 to $50 \mathrm{~Gy}$ in 1.8 to $2 \mathrm{~Gy}$ daily fractions. Patients having undergone BCS are advised tumor bed boost $(1.5-2 \mathrm{~cm}$ volume around surgical cavity) of 10 to $16 \mathrm{~Gy}$ in 5 to 8 daily fractions with electrons, in addition to whole breast irradiation (WBR) delivered via tangent fields using high energy $\mathrm{X}$-rays. Many centers now employ hypofractionation where a smaller total dose of RT is delivered in larger fractions over fewer days with encouraging results from the START-A and START-B trials. ${ }^{47,48}$

\section{Adjuvant Radiation Therapy to Internal Mammary Lymph Nodal Basin}

Ipsilateral IMLN metastases occur in 4 to $9 \%$ of axillary node-negative patients, and 16 to $65 \%$ of axillary node-positive patients. ${ }^{36}$ Though routine surgical dissection of the IMLN was abandoned owing to lack of survival benefit, routine irradiation of the IMLN was also abandoned in view of increased morbidity. As the benefits of PMRT were established, interest in IMLN irradiation was rekindled. A meta-analysis by Budach et $\mathrm{al}^{49}$ has shown that regional RT to the IMLN and medial SCLN significantly improves the DFS, distant metastases free survival (DMFS), and OS in stages I to III BC.

\section{Adjuvant Hormonal Therapy}

HT (tamoxifen in premenopausal or postmenopausal women, and aromatase inhibitors [AI] in postmenopausal women ${ }^{50}$ ) has contributed to improvements in DFS and OS significantly in hormone-positive BC. Debate regarding the apt duration for these treatments is ongoing. HT is traditionally given for 5 years but its extended usage has been explored as well. Exposure to AIs at some point during HT has shown improvements in DFS and may be considered in patients on tamoxifen after completion of the recommended 5 years of tamoxifen, for 2 more years. The extended duration of tamoxifen to 10 years has also shown significant benefit. ${ }^{51,52}$ Both classes of drugs possess unique side-effect profiles which the patient must be counseled about, and appropriate countermeasures must be taken.

\section{Adjuvant Ovarian Suppression}

Two landmark trials have reported a significant reduction in risk of recurrence with the addition of ovarian suppression to tamoxifen in younger hormone receptor-positive premenopausal women ( $\leq 40$ years) with high-risk features. ${ }^{53,54}$ Ovarian suppression may be achieved either medically by administering GnRH analogs (e.g., goserelin and leuprolide) or surgically by an oophorectomy, resulting in premature onset of menopause. Such patients may benefit from having bone-modifying agents like denosumab or bisphosphonates added to their treatment. ${ }^{55}$ It has a protective effect against loss of bone density and has been shown to reduce the risk of recurrence. ${ }^{56}$

\section{Follow-up in Breast Cancer Survivors}

The American Society of Clinical Oncology (ASCO) guidelines suggest that following completion of active $\mathrm{BC}$ treatment, patients are followed-up once in every 3 months in the first 3 years, 6 monthly in the fourth and fifth years, and annually thereafter with clinical breast examination (CBE) and annual MMG to assess for any recurrence and side-effects of treatment. ${ }^{57}$ Routine blood investigations and extensive imaging to detect distant metastases are not warranted, unless the patient complains of symptoms arousing 
suspicion of recurrence or if clinically indicated, since there is no evidence of positive impact on OS by early detection of locoregional/distant metastasis.

\section{Tailoring Locally Advanced Breast Cancer Treatment in the Indian Setting}

In a low-middle income country, like India, with resource constraints, the value of a treatment is also determined by its cost effectiveness. Cancer care poses a major financial burden on health care budgets and is a drain on the affected individual/family's income, especially among those without health insurance. This is a concern at every step of LABC treatment at initial diagnosis while planning NST, surgery, and/or, adjuvant locoregional treatments.

Close to half of the patients with BC in India present to the clinic at advanced stages at presentation. In developing and heavily populated countries like India, annual government screening programs which ensure early detection of the disease is a pipe dream considering the tremendous financial investment involved. This may be tackled by allocating finances toward educating the public about SBE, benefits of early detection, and training of community health care workers in CBE. A study by Okonkwo et al reported that annual CBE from ages 40 to 60 was predicted to be nearly as efficacious as biennial MMG for reducing BC mortality while incurring only half of the net costs. ${ }^{58}$ This is further reiterated by the hot-off-the-printing-press results of a large, 20-year-long RCT conducted in Mumbai, India, which demonstrated a 30\% significant reduction in $\mathrm{BC}$ mortality in women aged more than 50 years when screened 2 yearly by $\mathrm{CBE}$ by trained primary health workers. ${ }^{59}$ Early detection would strengthen clinicians with more conservative options of treatments in their armamentarium, so that patients may be offered conservative modalities of surgical intervention such as sentinel lymph node biopsy, BCT, etc., therefore, reducing costs toward treating morbidity incurred among the treated population and improving quality of life (QOL). Detecting BC in the early stages may also help save costs on prevented palliative care.

Of the many choices of systemic therapies available in our kitty, one must choose the drug which will have the least financial impact on the patient. The treatment of HER2-positive BC, for instance, shows more than $50 \%$ benefit in DFS with the addition of trastuzumab to adjuvant chemotherapy for a duration of 1 year but is unavailable to many owing to its prohibitively high costs. A solution would be to incorporate a shorter 9-week regimen of the drug in the adjuvant setting which has shown to offer equivalent benefit. ${ }^{60}$ Using biosimilars, which are drugs that are unidentical to the original molecule but have proven similar efficacy at one-fourth of the cost of the original molecule, is another viable alternative. ${ }^{61,62}$ Adoption of dose-dense regimens to deliver chemotherapy and hypofractionation in adjuvant RT delivery improves cost effectiveness by reducing treatment duration. Concurrent chemoradiation is being studied by a few centers worldwide, including a regional cancer institute in India and preliminary evidence suggests improved rates of
pCR using concurrent taxanes with RT ${ }^{63}$ A study by Adams et al demonstrated that this improved rate of pCR translates into improved survival outcomes. ${ }^{64}$ This may also improve cost effectiveness, if approved by expert consensus to become standard of care. It would be prudent to plan oophorectomy and mastectomy in the same setting in high-risk hormone receptor-positive women who have completed their family, instead of initiating prolonged monthly or 3 monthly dosing of expensive GnRH analogs or oophorectomy at a later stage.

Breast reconstruction (immediate/delayed) is being increasingly offered to women undergoing mastectomy, factoring in body image and QOL indices. Most insurance schemes in developing nations do not cover the costs of breast reconstruction, since it is viewed more as a cosmetic procedure rather than an essential part of postmastectomy rehabilitation. This dramatically increases out-of-pocket costs for the patients, hence being an unaffordable option. It would be pertinent for the various government and private operators to recognize the contribution of breast reconstruction toward the improvement of QOL, body image, its relevance in cancer care, and consider including it in their reimbursement schemes.

\section{Conclusion}

LABC is best tackled with multimodal management, implementing the strengths of systemic and locoregional therapies. BC treatment is individualized based on disease biology, patient characteristics, accessibility, and choice. It is essential to assess disease response at every stage of treatment. Surgical options for LABC management now extend beyond the traditionally practiced radical extirpations to more conservative approaches, but patient selection is a key. Adjuvant RT adds significant therapeutic benefit. More studies are warranted to explore its utility, concurrent with NST, given a few encouraging reports. Periodic follow-up of BC patients, as suggested by various international treatment guidelines is of immense importance to detect metastases. LABC treatment poses a great financial burden on patients and their families. Efforts must be made by the government and health care professionals alike, to propagate "breast-awareness" in society. Early detection of $\mathrm{BC}$ widens the spectrum of available treatment options, improves overall outcomes, and is less resource intensive. Considering the steadily increasing incidence of $\mathrm{BC}$, it would be prudent for society to recognize the importance of prioritizing health, make appropriate lifestyle modifications, and invest in private or government health insurance schemes.

\section{Conflict of Interest}

None declared.

\section{References}

1 International Agency for Research on Cancer; World Health Organization. Brest. Available at: https://gco.iarc.fr/today/ data/factsheets/cancers/20-Breast-fact-sheet.pdf. Accessed December 29, 2020 
2 Malvia S, Bagadi SA, Dubey US, Saxena S. Epidemiology of breast cancer in Indian women. Asia Pac J Clin Oncol 2017;13(4):289-295

3 Agarwal G, Ramakant P. Breast cancer care in India: the current scenario and the challenges for the future. Breast Care Basel 2008;3(1):21-27

4 Amim MB, Edge SG, Greene F, et al. eds. AJCC Cancer Staging Manual. Switzerland: Springer; 2017

5 Jaiyesimi IA, Buzdar AU, Hortobagyi G. Inflammatory breast cancer: a review. J Clin Oncol 1992;10(6):1014-1024

6 Lerebours F, Bieche I, Lidereau R. Update on inflammatory breast cancer. Breast Cancer Res 2005;7(2):52-58

7 Gradishar W, Moran M, Abraham J, et al. 2021. NCCN Guidelines ${ }^{\circ}$ Insights: Breast Cancer, Version 4.2021: Featured Updates to the NCCN Guidelines. [online] JNCCN. Available at: $<$ https://jnccn.org/view/journals/jnccn/19/5/article-p484. xml> [Accessed 7 July 2021].

8 Buzdar AU, Singletary SE, Booser DJ, Frye DK, Wasaff B, Hortobagyi GN. Combined modality treatment of stage III and inflammatory breast cancer. M.D. Anderson Cancer Center experience. Surg Oncol Clin N Am 1995;4(4):715-734

9 Bonadonna G, Valagussa P, Moliterni A, Zambetti M, Brambilla C. Adjuvant cyclophosphamide, methotrexate, and fluorouracil in node-positive breast cancer: the results of 20 years of follow-up. N Engl J Med 1995;332(14):901-906

10 Peto R, Davies C, Godwin J, et al. Early Breast Cancer Trialists' Collaborative Group (EBCTCG). Comparisons between different polychemotherapy regimens for early breast cancer: meta-analyses of long-term outcome among 100,000 women in 123 randomised trials. Lancet 2012;379(9814) :432-444

11 Moore HCF, Unger JM, Phillips K-A, et al. Final analysis of the prevention of early menopause study (POEMS)/SWOG Intergroup S0230. J Natl Cancer Inst 2019;111(2):210-213

12 Rastogi P, Anderson SJ, Bear HD, et al. Preoperative chemotherapy: updates of National Surgical Adjuvant Breast and Bowel Project Protocols B-18 and B-27. J Clin Oncol 2008;26(5):778-785

13 Citron ML, Berry DA, Cirrincione C, et al. Randomized trial of dose-dense versus conventionally scheduled and sequential versus concurrent combination chemotherapy as postoperative adjuvant treatment of node-positive primary breast cancer: first report of Intergroup Trial C9741/Cancer and Leukemia Group B Trial 9741. J Clin Oncol 2003;21(8):1431-1439

14 Hussain N, Said AS, Khan Z. Safety assessment of neoadjuvant pertuzumab combined with trastuzumab in nonmetastatic HER2-positive breast cancer in postmenopausal elderly women of South Asia. Int J Breast Cancer 2018;2018:6106041

15 Slamon D, Eiermann W, Robert N, et al. Breast Cancer International Research Group. Adjuvant trastuzumab in HER2-positive breast cancer. $\mathrm{N}$ Engl J Med 2011;365(14):1273-1283

16 von Minckwitz G, Huang C-S, Mano MS, et al. KATHERINE Investigators. Trastuzumab emtansine for residual invasive HER2-positive breastcancer.NEnglJMed 2019;380(7):617-628

17 Masuda N, Lee S-J, Ohtani S, et al. Adjuvant capecitabine for breast cancer after preoperative chemotherapy. N Engl J Med 2017;376(22):2147-2159

18 Spring LM, Gupta A, Reynolds KL, et al. Neoadjuvant endocrine therapy for estrogen receptor-positive breast cancer: a systematic review and meta-analysis. JAMA Oncol 2016;2(11):1477-1486

19 Halsted WS. I. The results of operations for the cure of cancer of the breast performed at the Johns Hopkins Hospital from June, 1889, to January, 1894. Ann Surg 1894;20(5):497-555

20 McWHIRTER R. The value of simple mastectomy and radiotherapy in the treatment of cancer of the breast. $\mathrm{Br}$ J Radiol 1948;21(252):599-610
21 Fisher B. Laboratory and clinical research in breast cancer-a personal adventure: the David A. Karnofsky memorial lecture. Cancer Res 1980;40(11):3863-3874

22 Fisher B, Jeong J-H, Anderson S, Bryant J, Fisher ER, Wolmark N. Twenty-five-year follow-up of a randomized trial comparing radical mastectomy, total mastectomy, and total mastectomy followed by irradiation. N Engl J Med 2002;347(8):567-575

23 Fisher B, Anderson S, Bryant J, et al. Twenty-year follow-up of a randomized trial comparing total mastectomy, lumpectomy, and lumpectomy plus irradiation for the treatment of invasive breast cancer. N Engl J Med 2002;347(16):1233-1241

24 Veronesi U, Cascinelli N, Mariani L, et al. Twenty-year follow-up of a randomized study comparing breast-conserving surgery with radical mastectomy for early breast cancer. $\mathrm{N}$ Engl J Med 2002;347(16):1227-1232

25 Veronesi U, Bonadonna G, Zurrida S, et al. Conservation surgery after primary chemotherapy in large carcinomas of the breast. Ann Surg 1995;222(5):612-618

26 Scholl SM, Fourquet A, Asselain B, et al. Neoadjuvant versus adjuvant chemotherapy in premenopausal patients with tumours considered too large for breast conserving surgery: preliminary results of a randomised trial: S6. Eur J Cancer 1994;30A(5):645-652

27 Mauriac L, MacGrogan G, Avril A, et al. Institut Bergonié Bordeaux Groupe Sein (IBBGS). Neoadjuvant chemotherapy for operable breast carcinoma larger than $3 \mathrm{~cm}$ : a unicentre randomized trial with a 124-month median follow-up. Ann Oncol 1999;10(1):47-52

28 Parmar V, Krishnamurthy A, Hawaldar R, et al. Breast conservation treatment in women with locally advanced breast cancer experience from a single centre. Int J Surg 2006;4(2):106-114

29 Chen AM, Meric-Bernstam F, Hunt KK, et al. Breast conservation after neoadjuvant chemotherapy: the MD Anderson cancer center experience. J Clin Oncol 2004;22(12):2303-2312

30 Zhou X, Li Y. Local recurrence after breast-conserving surgery and mastectomy following neoadjuvant chemotherapy for locally advanced breast cancer - a meta-analysis. Breast Care Basel 2016;11(5):345-351

31 Mamounas EP, Anderson SJ, Dignam JJ, et al. Predictors of locoregional recurrence after neoadjuvant chemotherapy: results from combined analysis of National Surgical Adjuvant Breast and Bowel Project B-18 and B-27. J Clin Oncol 2012;30(32):3960-3966

32 Houssami N, Macaskill P, Marinovich ML, Morrow M. The association of surgical margins and local recurrence in women with early-stage invasive breast cancer treated with breast-conserving therapy: a meta-analysis. Ann Surg Oncol 2014;21(3):717-730

33 Silverstein MJ, Savalia N, Khan S, Ryan J. Extreme oncoplasty: breast conservation for patients who need mastectomy. Breast J 2015;21(1):52-59

34 Koppiker CB, Noor AU, Dixit S, et al. Extreme oncoplastic surgery for multifocal/multicentric and locally advanced breast cancer. Int J Breast Cancer 2019;2019:4262589

35 Veronesi U, Cascinelli N, Bufalino R, et al. Risk of internal mammary lymph node metastases and its relevance on prognosis of breast cancer patients. Ann Surg 1983;198(6):681-684

36 Handley RS, Thackray AC. Invasion of internal mammary lymph nodes in carcinoma of the breast. BMJ 1954;1(4853):61-63

37 Urban JA. Extended radical mastectomy for breast cancer. Am J Surg 1963;106:399-404

38 Lacour J, Bucalossi P, Cacers E, et al. Radical mastectomy versus radical mastectomy plus internal mammary dissection. Five-year results of an international cooperative study. Cancer 1976;37(1):206-214

39 Sugarbaker ED. Extended radical mastectomy: its superiority in the treatment of breast cancer. JAMA 1964;187(2):96-99 
40 Veronesi U, Valagussa P. Inefficacy of internal mammary nodes dissection in breast cancer surgery. Cancer 1981;47(1):170-175

41 Overgaard M, Hansen PS, Overgaard J, et al. Postoperative radiotherapy in high-risk premenopausal women with breast cancer who receive adjuvant chemotherapy. Danish Breast Cancer Cooperative Group 82b Trial. N Engl J Med 1997;337(14):949-955

42 Overgaard M, Jensen MB, Overgaard J, et al. Postoperative radiotherapy in high-risk postmenopausal breast-cancer patients given adjuvant tamoxifen: Danish Breast Cancer Cooperative Group DBCG 82c randomised trial. Lancet 1999;353(9165) :1641-1648

43 Ragaz J, Olivotto IA, Spinelli JJ, et al. Locoregional radiation therapy in patients with high-risk breast cancer receiving adjuvant chemotherapy: 20-year results of the British Columbia randomized trial. J Natl Cancer Inst 2005;97(2):116-126

44 Clarke M, Collins R, Darby S, et al. Early Breast Cancer Trialists' Collaborative Group (EBCTCG). Effects of radiotherapy and of differences in the extent of surgery for early breast cancer on local recurrence and 15-year survival: an overview of the randomised trials. Lancet 2005;366(9503):2087-2106

45 Recht A, Comen EA, Fine RE, et al. Postmastectomy radiotherapy: an American Society of Clinical Oncology, American Society for Radiation Oncology, and Society of Surgical Oncology Focused Guideline update. Pract Radiat Oncol 2016;6(6):e219-e234

46 McGuire SE, Gonzalez-Angulo AM, Huang EH, et al. Postmastectomy radiation improves the outcome of patients with locally advanced breast cancer who achieve a pathologic complete response to neoadjuvant chemotherapy. Int J Radiat Oncol Biol Phys 2007;68(4):1004-1009

47 Bentzen SM, Agrawal RK, Aird EG, et al. START Trialists' Group. The UK Standardisation of Breast Radiotherapy (START) Trial A of radiotherapy hypofractionation for treatment of early breast cancer: a randomised trial. Lancet Oncol 2008;9(4):331-341

48 Bentzen SM, Agrawal RK, Aird EG, et al. START Trialists' Group. The UK Standardisation of Breast Radiotherapy (START) Trial B of radiotherapy hypofractionation for treatment of early breast cancer: a randomised trial. Lancet 2008;371(9618): 1098-1107

49 Budach W, Kammers K, Boelke E, Matuschek C. Adjuvant radiotherapy of regional lymph nodes in breast cancer - a meta-analysis of randomized trials. Radiat Oncol 2013;8:267

50 Mamounas EP, Bandos H, Lembersky BC, et al. A randomized trial of five years of letrozole versus placebo after aromatase inhibitor-based therapy: NRG Oncology/NSABP B-42. Lancet Oncol 2019;20(1):88-99

51 Davies C, Pan H, Godwin J, et al. Adjuvant Tamoxifen: Longer Against Shorter (ATLAS) Collaborative Group. Long-term effects of continuing adjuvant tamoxifen to 10 years versus stopping at 5 years after diagnosis of oestrogen receptor-positive breast cancer: ATLAS, a randomised trial. Lancet 2013;381(9869): 805-816
52 Gray RG, Rea D, Handley K, et al. aTTom: Long-term effects of continuing adjuvant tamoxifen to 10 years versus stopping at 5 years in 6,953 women with early breast cancer. JCO 2013;31(18(suppl):5-5

53 Francis PA, Regan MM, Fleming GF, et al. SOFT Investigators; International Breast Cancer Study Group. Adjuvant ovarian suppression in premenopausal breast cancer. N Engl J Med 2015;372(5):436-446

54 Pagani O, Regan MM, Walley BA, et al. TEXT and SOFT Investigators; International Breast Cancer Study Group. Adjuvant exemestane with ovarian suppression in premenopausal breast cancer. N Engl J Med 2014;371(2):107-118

55 Gnant M, Pfeiler G, Steger GG, et al. Austrian Breast and Colorectal Cancer Study Group. Adjuvant denosumab in postmenopausal patients with hormone receptor-positive breast cancer (ABCSG-18): disease-free survival results from a randomised, double-blind, placebo-controlled, phase 3 trial. Lancet Oncol 2019;20(3):339-351

56 Gnant M, Pfeiler G, Dubsky PC, et al. Austrian Breast and Colorectal Cancer Study Group. Adjuvant denosumab in breast cancer (ABCSG-18): a multicentre, randomised, double-blind, placebo-controlled trial. Lancet 2015;386(9992):433-443

57 Khatcheressian JL, Wolff AC, Smith TJ, et al. American Society of Clinical Oncology. American Society of Clinical Oncology 2006 update of the breast cancer follow-up and management guidelines in the adjuvant setting. J Clin Oncol 2006;24(31):5091-5097

58 Okonkwo QL, Draisma G, der Kinderen A, Brown ML, de Koning HJ. Breast cancer screening policies in developing countries: a cost-effectiveness analysis for India. J Natl Cancer Inst 2008;100(18):1290-1300

59 Mittra I, Mishra GA, Dikshit RP, et al. Effect of screening by clinical breast examination on breast cancer incidence and mortality after 20 years: prospective, cluster randomised controlled trial in Mumbai. BMJ 2021;372:n256

60 Joensuu H, Kellokumpu-Lehtinen P-L, Bono P, et al. FinHer Study Investigators. Adjuvant docetaxel or vinorelbine with or without trastuzumab for breast cancer. $\mathrm{N}$ Engl J Med 2006;354(8):809-820

61 Barbier L, Declerck P, Simoens S, Neven P, Vulto AG, Huys I. The arrival of biosimilar monoclonal antibodies in oncology: clinical studies for trastuzumab biosimilars. $\mathrm{Br}$ J Cancer 2019;121(3):199-210

62 Nelson KM, Gallagher PC. Biosimilars lining up to compete with Herceptin-opportunity knocks. Expert Opin Ther Pat 2014;24(11):1149-1153

63 Iyer P, Radhakrishnan V, Balasubramanian A, et al. Study of pathological complete response rate with neoadjuvant concurrent chemoradiation with paclitaxel in locally advanced breast cancer. Indian J Cancer 2020;57(4):428-434

64 Adams S, Chakravarthy AB, Donach M, et al. Preoperative concurrent paclitaxel-radiation in locally advanced breast cancer: pathologic response correlates with five-year overall survival. Breast Cancer Res Treat 2010;124(3):723-732 